\title{
EVALUATION OF PREGABALIN ADMINISTRATION PERIOPERATIVELY IN PAIN MANAGEMENT OF PATIENTS UNDERGOING HIP ARTHROPLASTY
}

E. Papaioannou , P. Doka, C. Schiza , H. Koziakas , A. Maniati , E. Papadopoulou. ,A. Lampadariou .

Laikpn General Hospital of Athens. Greece

Department of Anaesthesia and Pain Management,

\section{Background and Aims}

Goal of this study was to evaluate postoperative pain using pregabalin in patients undergoing hip arthroplasty,

\section{Methods}

56 patientsscheduled to total hip replacement, were studied. Patients were assigned into two groups (grouo A: 28 patients ( 8 male, 13 female) who were receiving pregabalin (100-300 mg per os) for at least two months preoperatively in a regular basis for their pain management, due to hip osteoarthritis, and group $B$ (control): 28 patients (13 male, 15 female), of similar age and weight of group $A$ who were receiving conventional pain killers for their condition preoperatively. In both groups' patients postoperatively the same analgesic protocol was applied: paracetamol $1 \mathrm{gr}$ iv $\mathrm{x} 4$, parecoxib $40 \mathrm{mg} \mathrm{x}$ 2 , and tramadol $50 \mathrm{mg}$ iv prn. Group A patients continued their pregabalin consumption, as preoperatively. Pain scores every 12 hrs during day of surgery and 3 first postoperative days, tramadol consumption in both groups and complications or side effects, were recorded.

\section{Results}

Pain according to $1-10$ VAS scale,was lower $(p<0.001)$ in group $A$ patients at all times of measurement $\quad(1.8 \pm 0.6 \quad$ vs $3.6 \pm 0.8, \quad 1.6 \pm 0.3$ vs $2.9 \pm 0.3$, $1.9 \pm 0.4$ vs $2.5 \pm 0.4,1.3 \pm 0.1$ vs $1.9 \pm 0.7, \quad 0.6 \pm 0.1$ vs $1.4 \pm 0,6$ $0.7 \pm 0.2$ vs $1.1 \pm 0.2,0.3 \pm 0.03$ vs $0.8 \pm 0.05$, and $0.4 \pm 0.01$ vs $0.7 \pm 0.1$ respectively). Mean tramadol consumption was lower in group $A$ patients on studied days $(100 \pm 22$ vs $250 \pm 88$, $150 \pm 35$ vs $300 \pm 42,50 \pm 23$ vs $250 \pm 49$, and 0 vs $200 \pm 21$ $\mathrm{mg} /$ day, on every day studied respectively $-p<0.001$ ). No side effects were observed in any time of study.

\section{Conclusions}

Pregabalin administration perioperatively. seems to reduce pain and opioid consumption in total hip arthroplasty 